\title{
PET Y PP USADO COMO MEDIOS DE CRECIMIENTO DE BIOPELÍCULA FIJA APLICADA AL TRATAMIENTO AEROBIO DE AGUAS RESIDUALES DOMÉSTICAS
}

\author{
B. $\mathrm{LAPO}^{1}$, M. MUÑOZ ${ }^{2}$, H. ROMERO ${ }^{1}, \mathrm{M} \mathrm{POZO}^{2}, \mathrm{H}^{\text {AYALA }}{ }^{1}$ \\ ${ }^{1}$ Universidad Técnica de Machala, Facultad de Ciencias Químicas y de la Salud \\ ${ }^{2}$ Escuela Politécnica Nacional, Facultad de Ingeniería Civil y Ambiental \\ E-mail para contacto: byronglc@gmail.com
}

\begin{abstract}
RESUMEN - Se evaluó el desempeño de plásticos reciclables como Polietileno Tereftalato PET y Polipropileno PP como medios de soporte para el crecimiento de biopelícula en reactores aerobios de $2.8 \mathrm{~L}$ de lecho fijo para el tratamiento de agua residual doméstica. Estos materiales fueron evaluados durante 300 días. Los reactores con PET contenían pedazos de $5 \mathrm{~cm} \times 1 \mathrm{~cm}$, mientras que los $\mathrm{PP}$ con tiras de $80 \mathrm{~cm} \times 5 \mathrm{~cm}$. Como sustrato se utilizó agua residual del río Machángara (Quito-Ecuador), cuyo DQOs fué de $200 \mathrm{mg} / \mathrm{L}$, a la que se fortificó con melaza entre 795 a $1420 \mathrm{mg} / \mathrm{L}$. El área específica para los materiales PET y PP fue de $654,62 \mathrm{~m} 2 / \mathrm{m} 3$ y $882,5 \mathrm{~m} 2 / \mathrm{m} 3$ respectivamente. Durante este tiempo la carga orgánica expresada en gramos de DQOs fue para los reactores con material PET de 16,82 g y 17,95 g DQOs para aquellos con PP. Se logró eficiencias máximas de remoción de DQOs del $95 \%$ y $96 \%$ para PET y PP respectivamente, siendo este último el mejor dado su mayor porcentaje de remoción alcanzado en menor tiempo.
\end{abstract}

\section{INTRODUCCION}

La limitación de espacios para instalar plantas para tratamiento de aguas residuales obliga a desarrollar nuevas tecnologías para incrementar la capacidad de los sistemas depuradores por unidad de área construida. Una alternativa son los sistemas que utilizan el crecimiento de biopelículas (Chen I, 2000).

Los reactores de biopelícula tanto de lecho fijo, como móvil fueron desarrollados a finales de los años 1980 y principios de 1990 (Odegaard H., 1999). Hoy en día existen más de 400 instalaciones alrededor del mundo en sectores tanto municipales como industriales. Las ventajas de un proceso FBBR (fixed bed biofilm reactor) como MBBR (moving bed biofilm reactor) sobre los métodos tradicionales como por ejemplo de lodos activados incluyen: mejor transferencia de oxígeno, cortos tiempos de retención hidráulica, altas tasas de carga orgánica, alta tasa de nitrificación y gran área específica disponible para transferencia de masa (Sombatsompop K., 2006) y (Chan Y., 2009).

La utilización de medios de soporte de bajo precio y de fácil disponibilidad en nuestro entorno para la utilización en reactores de biopelícula aplicado al tratamiento de aguas residuales urbanas, es 
de vital importancia para la aplicación tecnológica de este tipo de sistemas de tratamiento, gracias a que prestan las siguientes ventajas.

- Capacidad de cumplir con objetivos de tratamiento similares a los obtenidos con lodos activados, con respecto a remoción de DBO5 y nitrógeno, pero en volúmenes de tratamiento más pequeño. (Andreottola G., 2000)

- Son procesos continuos que no requieren operación especial en lo que respecta al control de espesor de biomasa. (James P. McQuarrie, 2011)

- Capacidad de prueba y uso de materiales residuales como medio de soporte tales como plásticos provenientes de botellas usadas de bebidas de consumo o cualquier otro material que pueda retener biomasa, lo que beneficia doblemente al ambiente, ya que por una parte se reduciría una cantidad de plástico de los residuos sólidos y por otra parte serían ayuda en el tratamiento de aguas residuales.

En la actualidad el uso de éste sistema de tratamiento en Ecuador es poco frecuente, y los medios de soporte más utilizados son piedras, cascajos y otros materiales cuyas limitaciones tecnológicas han sido descritas en varias investigaciones. Por otro lado a nivel internacional se han desarrollado medios de soporte con las características suficientes para lograr concentraciones aceptables de biofilm, pero el costo local es el principal limitante al momento del uso de estos materiales sintéticos.

En los sistemas de tratamiento basados en biopelícula, la mayoría de los microorganismos tienden a unirse entre sí, a adherirse a diversas superficies sólidas, a reproducirse y a crecer mediante la producción de polisacáridos extracelulares (PEC). Los biofilms pueden formarse en superficies vivas o no vivas y pueden prevalecer en medios naturales, industriales $\mathrm{u}$ hospitalarios. Las bacterias se agrupan en colonias con el objeto de buscar protección ante posibles condiciones adversas del medio.

Los microorganismos formadores de biopelícula poseen algunas ventajas sobre los microorganismos suspendidos como: mayor persistencia dentro del sistema; mayores tasas de crecimiento; mayor incremento en la actividad metabólica; y mayor resistencia a la toxicidad.

La composición de una biopelícula está determinada por tres factores principalmente: por la composición del medio, la expansión volumétrica de la biomasa y por el transporte de sustratos hacia el interior de la biopelícula (Costerton, 1995). Acorde a (Senthilnathan \& Ganczarczyk, 1990), la concentración de biomasa dentro de un sistema de lodos activados se mantiene en el rango de 700 $\mathrm{mg} / \mathrm{L}$ a $2500 \mathrm{mg} / \mathrm{L}$ (expresados en términos de Solidos Suspendidos Volátiles de licor mixto (SSVLM)), mientras que en biofiltros de goteo, la biomasa adherida esta en rangos de $2000 \mathrm{mg} / \mathrm{L} \mathrm{a}$ $100000 \mathrm{mg} / \mathrm{L}$ de SSVLM.

Los tratamientos de aguas residuales por película biológica soportada, tanto en sistemas fijos como móviles utilizan medios plásticos inertes que provean alta superficie específica para cargar la 
biomasa que trata el agua. La principal función del material de soporte (relleno) es ofrecer una gran superficie que favorezca la adhesión de los microorganismos y la formación de una película biológica activa, al mismo tiempo que asegure el atrapamiento, en los espacios vacíos del lecho, de los flóculos microbianos no adheridos (Baecheler, et al. 2001). Las características del medio de soporte de la biopelícula son relevantes durante las primeras etapas de formación de ésta, ya que influyen tanto en la velocidad de formación como en la distribución de las especies dentro de la biopelícula.

Las principales finalidades del medio de soporte, son: mejorar el contacto entre el sustrato y los sólidos biológicos contenidos en el reactor, permitir la acumulación de gran cantidad de biomasa, facilitar un flujo uniforme en el reactor, evitar que los sólidos sean arrastrados por fuera del sistema de tratamiento y, actuar como un dispositivo para separar los sólidos de los gases.

\subsection{Materiales y Métodos}

Se evaluó por triplicado dos medios de soporte plásticos provenientes de materiales reciclables en dos series de reactores aerobios de biopelícula en lecho fijo operados de forma discontinua. El tiempo de corrida de los reactores fue de 330 días, tiempo en el cual se estableció la máxima cantidad de biomasa que fue adherida a los medios de soporte, además la eficiencia de remoción de DQOs fue evaluada.

\subsubsection{Reactores}

La figura 1 muestra una fotografía de los reactores en funcionamiento, mientras que en la figura 2 se puede apreciar una ilustración de los reactores montados.

La tabla 1 y tabla 2 muestra las características de los reactores con los que fueron montados, y las condiciones a las que fueron operados respectivamente.

\subsubsection{Sustrato}

El sustrato empleado fue agua residual doméstica proveniente de la ciudad de Quito-Ecuador (DQOs promedio igual $200 \mathrm{mg} / \mathrm{L}$ ), la cual fue fortificada con melaza para alcanzar DQO soluble (DQOs) en el rango de $795 \mathrm{mg} / \mathrm{L}$ a $1420 \mathrm{mg} / \mathrm{L}$ con el que fueron alimentados progresivamente los modelos durante los ensayos.

\subsubsection{Medios de Soporte}

Se estudió el crecimiento de biopelícula en dos medios de soporte: Polietileno Tereftalato (PET) y Polopropileno (PP) proveniente de botellas de plástico usadas y tela de saquillo respectivamente; acorde a cada medio de soporte se trabajó por triplicado para poder evaluar diferencias en los rendimientos de cada uno, configurándose de esta manera dos grupos de tres reactores. Estos fueron seleccionados de acuerdo a la disponibilidad en el mercado.

El material PET empleado como medio de soporte que es mostrado en la figura 3 , proveniente 


\section{9 a 22 de outubro de 2014 \\ Florianópolis/SC}

de botellas de bebidas gaseosas y no gaseosas de consumo humano usadas fueron cortados en unidades de aproximadamente $1 \times 8 \mathrm{~cm}$; por su parte el otro material de soporte PP en estudio es proveniente de malla o tela de saquillo sintético, éste fue cortado en tiras de aproximadamente $85 \mathrm{~cm}$ de largo por $5 \mathrm{~cm}$ de ancho y usándose aproximadamente 17 a 19 tiras por cada reactor, dispuestos en cuatro pisos o niveles como se puede apreciar en la figura 4.

\subsubsection{Ensayos}

El pH, temperatura, cantidad de luz expuesta no fue modificada durante los experimentos. Los parámetros: DQO soluble (DQOs), Sólidos Suspendidos Volátiles (SSV) fueron evaluados constantemente para el estudio de su comportamiento. Nutrientes como Fósforo (P-total) y Nitrógeno total (N-total) fueron analizados en el arranque de los reactores con el fin de establecer si existía la cantidad necesaria de éstos para el desenvolvimiento normal de las reacciones bioquímicas dentro del reactor.

\subsection{Resultados y Discusión}

\subsection{1. Área específica}

En el caso de los dos materiales evaluados en la presente investigación PET y PP, éstos se pueden considerar valores aceptables o competitivos con los medios de soporte manufacturados ya que tienen $654 \mathrm{~m}^{2} / \mathrm{m}^{3}$ y $882 \mathrm{~m}^{2} / \mathrm{m}^{3}$ respectivamente. Respecto a otros medios de soporte manufacturados o pre-fabricados; se han reportado áreas específicas de éstos figuran entre 402 a 1200

$\mathrm{m}^{2} / \mathrm{m}^{3}$. (McQuarrie J. et al. 2011). Se nota además que el promedio el material B evaluado presenta mejor área específica o mayor disponibilidad de área para el crecimiento de biopelícula.

\subsubsection{Remoción de DQOs}

Los reactores fueron operados añadiéndose cargas entre $795 \mathrm{mg} / \mathrm{L}$ y $1420 \mathrm{mg} / \mathrm{L}$ de DQOs, lo que se representa en la figura 5, y que representó cargas másicas totales a lo largo de la experimentación de DQOs entre 16,67 a 18,34 g de DQOs para cada reactor. La figura muestra las concentraciones de DQO añadidas a los reactores durante los 330 días de operación.

La figura 6 muestra los promedios de remoción de DQOs de los dos medios de soporte evaluados. Las líneas PET-A, PP-A corresponden a la concentración de DQOs en los reactores luego de ser abastecidos para los materiales de soporte PET y PP respectivamente, de igual manera las líneas PET-E y PP-E corresponden a la concentración en el efluente y las líneas \%EFICIENCIA PET y \%EFICIENCIA PP se han graficado en el eje secundario y representan el porcentaje de remoción de DQO para los dos materiales de soporte evaluados.

Por su parte la concentración de DQOs en el efluente se mantuvo con comportamientos similares en ambos casos aunque mejores eficiencias de remoción siempre fueron logrados por el PP evaluado. A partir del día 86 de experimentación la eficiencia de remoción alcanzo el $65 \%$ para PP y luego de esto fue mejorando hasta lograr valores máximos de $96 \%$, evidenciándose siempre una mejor remoción que en el material de soporte de Polietileno Tereftalato. La eficiencia de remoción promedio 
luego del día 121 de estudio fue de $90 \%$ para PP y $87 \%$ para PET; presentando entonces mejor remoción el material B correspondiente a Polipropileno (malla de saquillo). Acorde a varios autores ( Li, 2013, Zhang et al. 1998), las tasas de remoción en sistemas de biopelícula móvil está entre 90$95 \%$, comportamiento similar a los de este trabajo.

\subsubsection{Biomasa adherida}

La figura 7 muestra la concentración de biomasa adherida a los medios de soporte expresada en gramos de Solidos Suspendidos Volátiles inmovilizados SSVi por metro cuadrado de material de soporte. En promedio el material de soporte A Polietileno tereftalato presento 1,023 gramos de biomasa medida como SSVi por metro cuadrado de material de soporte, mientras que el material B Polipropileno fue de 1,34 g SSVi/m2, siendo el material B quien presento más biomasa adherida y además acorde al análisis de eficiencia del reactor presenta mejores condiciones para este tipo de tratamiento. Otras investigaciones realizadas por diferentes autores donde se han evaluado sistemas tanto de biomasa suspendida en lecho fijo, como en lecho móvil, se reporta que por ejemplo Ahmet, 2008 consiguió cargas de biomasa de 9,36 a 13,12 g TSS/m2.

Los dos materiales de soporte evaluados PET y PP propiciaron el crecimiento de biopelícula en los reactores, por lo que pudieron remover la carga orgánica suministrada en lo que respecta a aguas residuales urbanas. El material PP (tela de saquillo plástico) obtuvo más fijación de biomasa que el material PET evaluado. La eficiencia de remoción de DQOs aunque al final de los experimentos fue igual, el material PP pudo remover en menor tiempo más DQOs.

\section{FIGURAS}

Figura 1. Reactores en funcionamiento

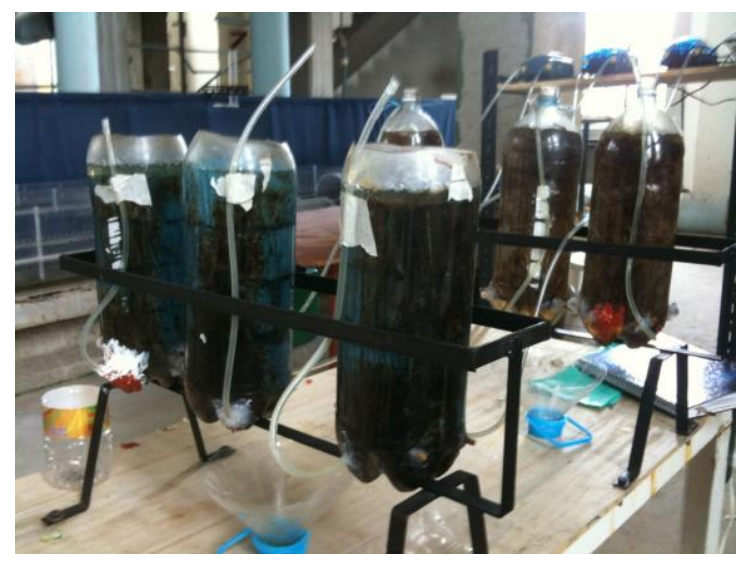




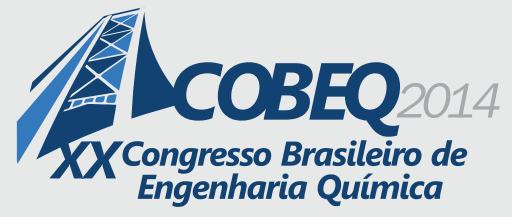

19 a 22 de outubro de 2014

Florianópolis/SC

Figura 2. Majonte experimental

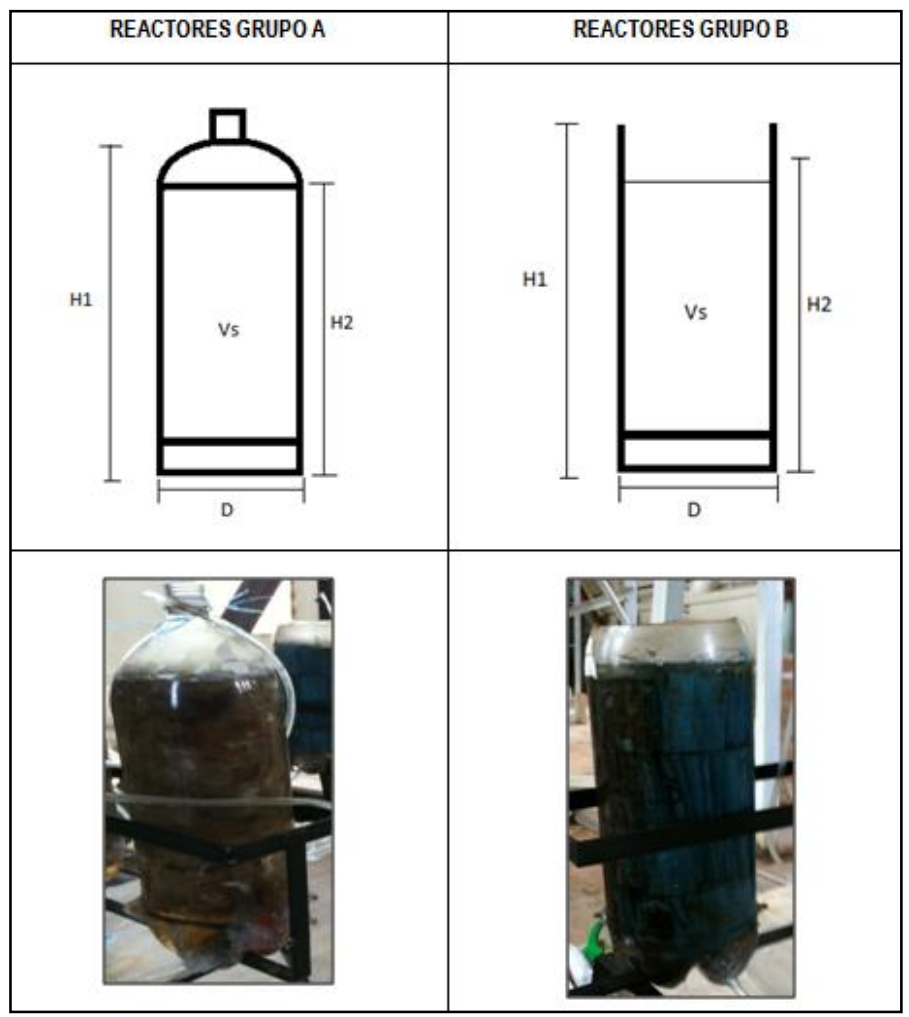

Figura 3. Material de polietileno tereftalato

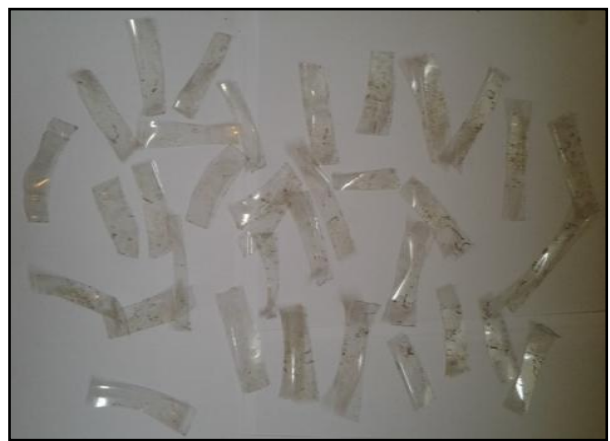

Figura 4. Material de Polipropileno

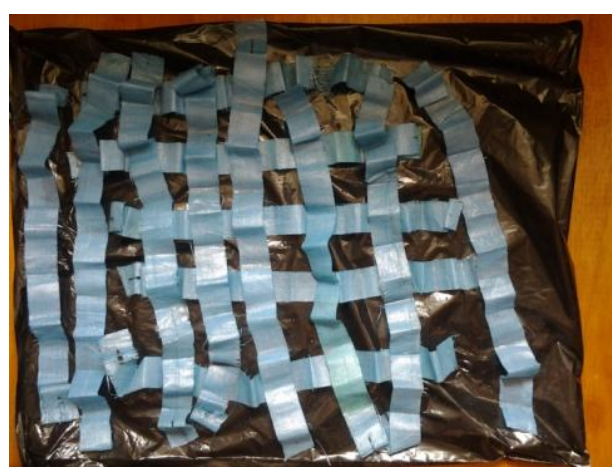


Figura 5. Concentración de DQO suministrada a los reactores

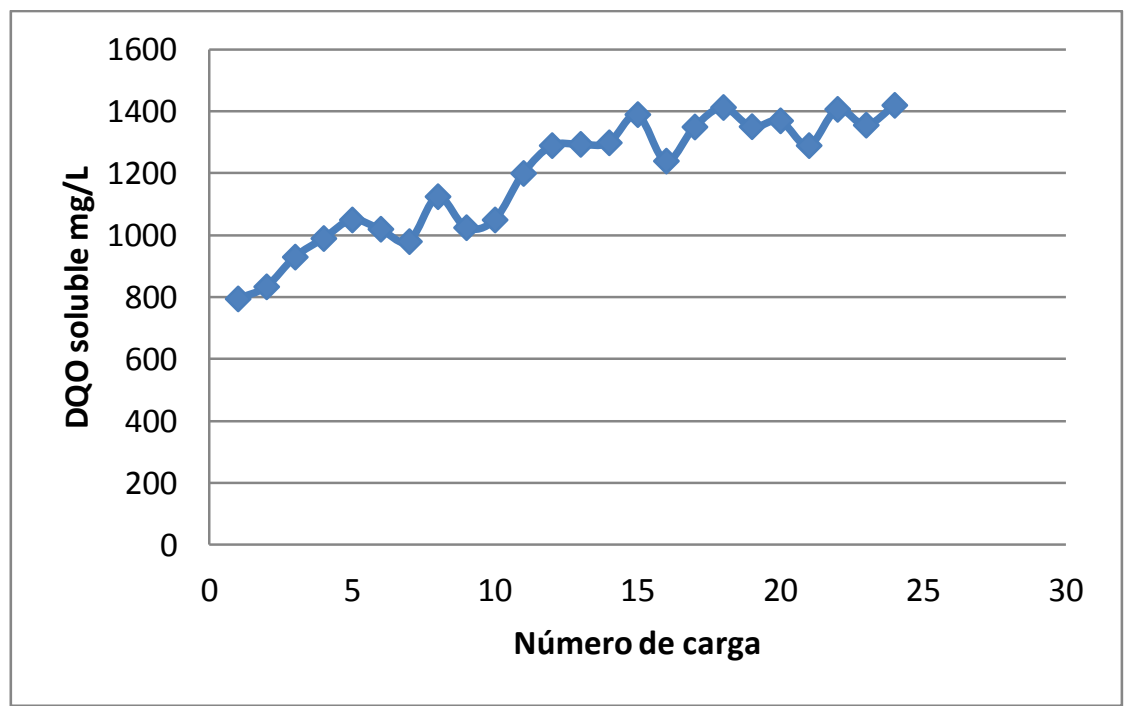

Figura 6. Remoción de DQOs promedio entre PET y PP

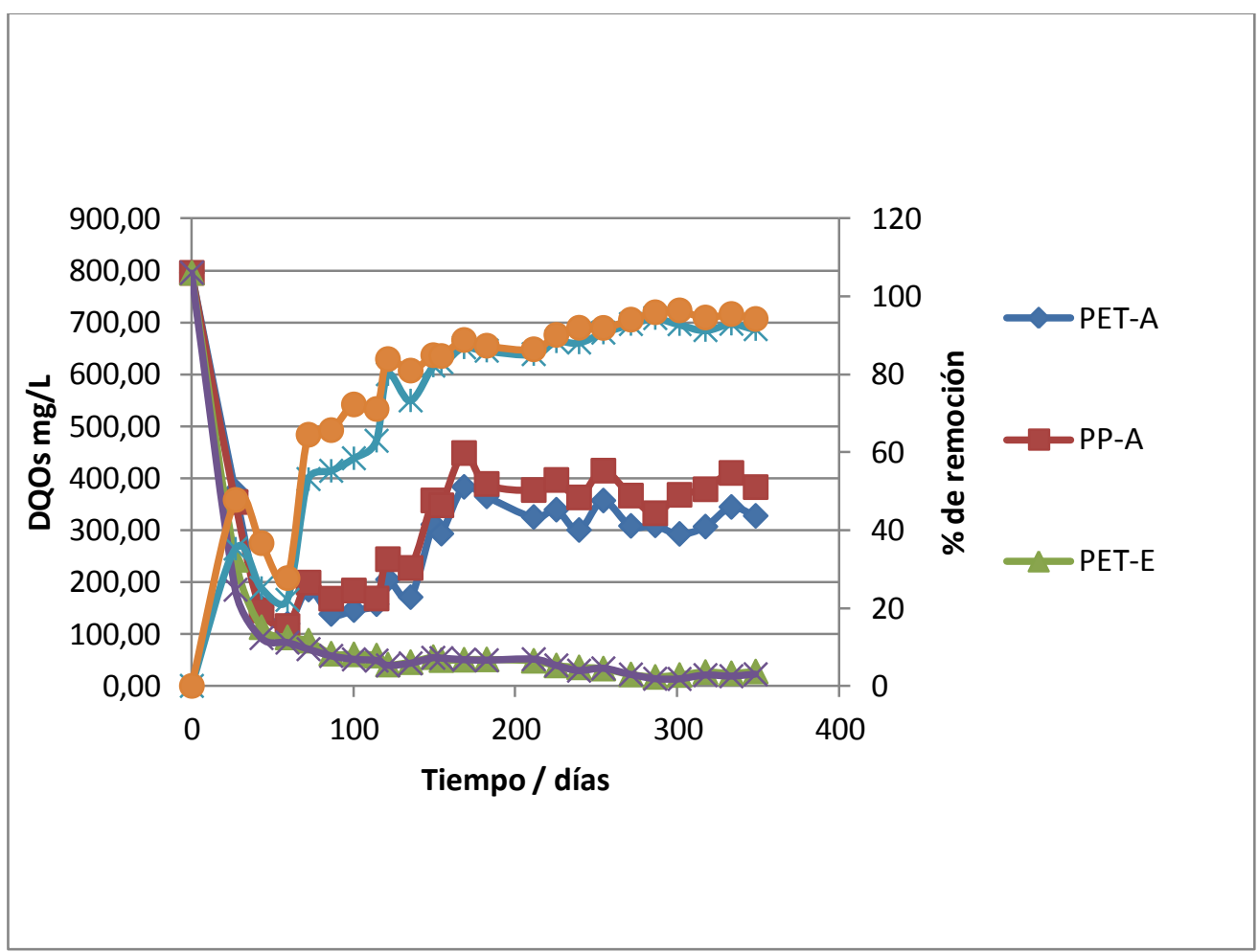


Figura 7. Biomasa adherida

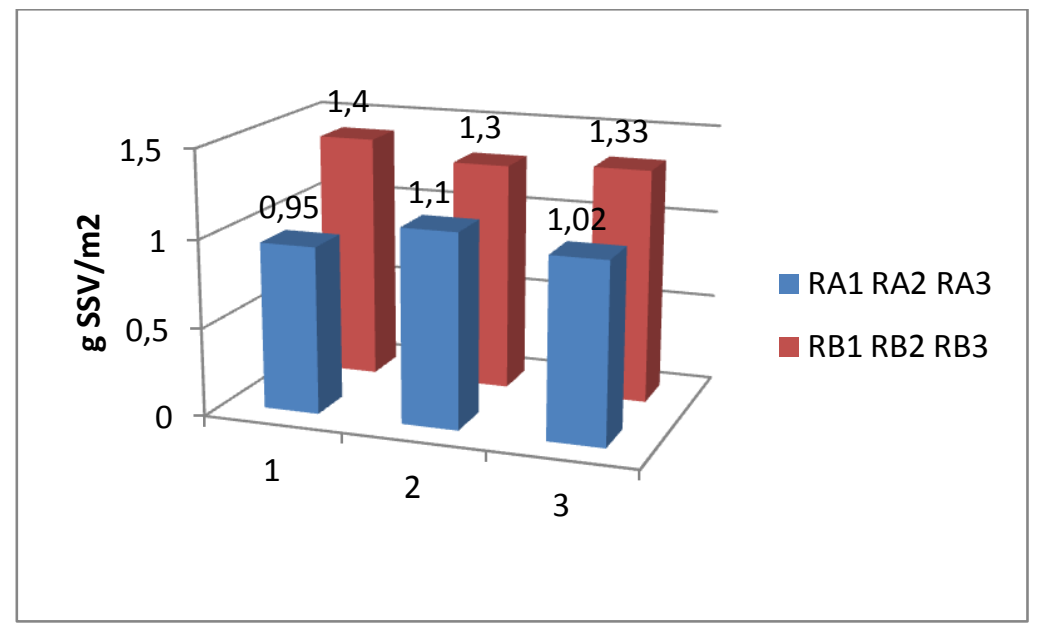

\section{TABLAS}

Tabla 1. Características físicas de los reactores.

\begin{tabular}{|l|l|l|l|}
\hline Característica & $\begin{array}{l}\text { Identificación en } \\
\text { el esquema }\end{array}$ & $\begin{array}{l}\text { GRUPO A - } \\
\text { PET }\end{array}$ & GRUPO B - PP \\
\hline Altura total del reactor $(\mathrm{cm})$ & $\mathrm{H} 1$ & 34,00 & 29,00 \\
\hline Altura de trabajo $(\mathrm{cm})$ & $\mathrm{H} 2$ & 29,50 & 26,50 \\
\hline Diámetro(cm) & $\mathrm{D}$ & 11 & 11 \\
\hline $\begin{array}{l}\text { Volumen disponible de trabajo } \\
\left(\mathrm{cm}^{2}\right)\end{array}$ & $\mathrm{Vt}$ & 2.803 .48 & 2.521 .90 \\
\hline $\begin{array}{l}\text { Volumen dispuesto al material de } \\
\text { soporte }\left(\mathrm{cm}^{2}\right)\end{array}$ & $\mathrm{Vs}$ & $2.185,76$ & 1.995 .70 \\
\hline Relación altura $:$ diámetro & $\mathrm{a}: \mathrm{d}$ & $3: 1$ & $3: 1$ \\
\hline Aireación: & & $\begin{array}{l}\text { Difusores tipo } \\
\text { pecera }\end{array}$ & $\begin{array}{l}\text { Difusores tipo } \\
\text { pecera }\end{array}$ \\
\hline
\end{tabular}

Tabla 2. Condiciones experimentales.

\begin{tabular}{|l|l|}
\hline VARIABLE & VALOR/CONDICION \\
\hline FLUJO DE AIRE $(\mathrm{L} / \mathrm{min})$ & 3 \\
\hline TEMPERATURA $\left({ }^{\circ} \mathrm{C}\right)$ & Ambiental Promedio:18 \\
\hline $\mathrm{Ph}$ & $\begin{array}{l}\text { No modificado durante la operación. Los } \\
\text { valores fluctuaron entre } 7.5-9.0\end{array}$ \\
\hline INTENSIDAD DE LUZ & $\begin{array}{l}\text { Reactores protegidos con lámina de } \\
\text { aluminio para que la luz no afecte } \\
\text { directamente en el proceso. }\end{array}$ \\
\hline MEDIO DE SOPORTE & $\begin{array}{l}\text { PET para grupo A de reactores. } \\
\text { PP para grupo B de reactores. }\end{array}$ \\
\hline
\end{tabular}




\section{NOMENCLATURA}

PP: Polipropileno

PET: Polietileno tereftalato

DQOs: Demanda química de oxígeno soluble

SSV: Sólidos suspendidos volátiles

DBO: Demanda Química de Oxígeno

\section{REFERÊNCIAS}

Chen I, C. F. Specificity of Microbial Activities in the Reductive Dechlorination of Chlorinated Benzenes. Wat. Environ. Res. 72, 675-679, 2000

Odegaard H., B. R. The development of the moving bed biofilm process form idea to commerical product. Eur. Wat Manage, 36, 1999.

Sombatsompop K., V. C. Evaluation of bio fouling phenomenon in suspended and attached growth membrane bioreactor systems. Desalination, 138-149, 2006

Chan Y., C. M. A review on anaerobic-aerobic treatment of industrial and municipal wastewater. Chemical Engineering, 1-18, 2009

Andreottola G., F. P. Experimental Comparison Between MBBR and Activated Sludge System for the Treatment of Municipal Wastewater. Water Sci. Technology, 375-382, 2000.

James P. McQuarrie, J. P. Moving Bed Biofilm Reactor Technology: Process Applications, Design, and Performance. Water Environment Research, 560-575, 2011.

Costerton, J. W.-S. Microbial biofilms. Annu. Rev. Microbiol., 711-745, 1995

Senthilnathan, P., \& Ganczarczyk, J. Application of biomass carriers in activated sludge process. Wastewater Treatment by Immobilzed Cells, 1990.

Baecheler, J., Zambrano, E., \& Urrutia, H. Estudio de la cinética de crecimiento de biopelículas bacterianas aplicadas en el tratamiento de riles. XIV Congreso de Ingeniería Sanitaria y Ambiental AIDIS. Santiago, 2001.

Li, A. Selecting the process. Biological wastewater treatment. (2013). 